\title{
BMJ Open Universal or targeted approach to prevent the transmission of extended- spectrum beta-lactamase-producing Enterobacteriaceae in intensive care units: a cost-effectiveness analysis
}

\author{
Lidia Kardaś-Słoma, ${ }^{1,2}$ Jean-Christophe Lucet, ${ }^{1,2,3}$ Anne Perozziello,, ${ }^{1,2}$ \\ Camille Pelat, ${ }^{1,2}$ Gabriel Birgand, ${ }^{1,2,3}$ Etienne Ruppé, ${ }^{4}$ Pierre-Yves Boëlle, ${ }^{5}$ \\ Antoine Andremont, ${ }^{4}$ Yazdan Yazdanpanah ${ }^{1,2,6}$
}

To cite: Kardaś-Słoma $\mathrm{L}$, Lucet J-C, Perozziello A, et al. Universal or targeted approach to prevent the transmission of extended-spectrum beta-lactamase-producing Enterobacteriaceae in intensive care units: a cost-effectiveness analysis. BMJ Open 2017;7:e017402. doi:10.1136/ bmjopen-2017-017402

- Prepublication history and additional material for this paper are available online. To view these files, please visit the journal (http://dx.doi.org/10. 1136/bmjopen-2017-017402).

Received 21 April 2017 Revised 1 August 2017 Accepted 10 August 2017

CrossMark

For numbered affiliations see end of article.

Correspondence to Dr Lidia Kardaś-Słoma; lidia.kardas@inserm.fr

\section{ABSTRACT}

Objective Several control strategies have been used to limit the transmission of multidrug-resistant organisms in hospitals. However, their implementation is expensive and effectiveness of interventions for the control of extendedspectrum beta-lactamase-producing Enterobacteriaceae (ESBL-PE) spread is controversial. Here, we aim to assess the cost-effectiveness of hospital-based strategies to prevent ESBL-PE transmission and infections.

Design Cost-effectiveness analysis based on dynamic, stochastic transmission model over a 1-year time horizon. Patients and setting Patients hospitalised in a hypothetical 10-bed intensive care unit (ICU) in a high-income country. Interventions Base case scenario compared with (1) universal strategies (eg, improvement of hand hygiene $(\mathrm{HH})$ among healthcare workers, antibiotic stewardship), (2) targeted strategies (eg, screening of patient for ESBL-PE at ICU admission and contact precautions or cohorting of carriers) and (3) mixed strategies (eg, targeted approaches combined with antibiotic stewardship).

Main outcomes and measures Cases of ESBL-PE transmission, infections, cost of intervention, cost of infections, incremental cost per infection avoided.

Results In the base case scenario, 15 transmissions and five infections due to ESBL-PE occurred per 100 ICU admissions, representing a mean cost of $€ 94792$. All control strategies improved health outcomes and reduced costs associated with ESBL-PE infections. The overall costs (cost of intervention and infections) were the lowest for $\mathrm{HH}$ compliance improvement from $55 \% / 60 \%$ before/after contact with a patient to $80 \% / 80 \%$.

Conclusions Improved compliance with $\mathrm{HH}$ was the most cost-saving strategy to prevent the transmission of ESBLPE. Antibiotic stewardship was not cost-effective. However, adding antibiotic restriction strategy to $\mathrm{HH}$ or screening and cohorting strategies slightly improved their effectiveness and may be worthy of consideration by decision-makers.

\section{INTRODUCTION}

The incidence of infection and colonisation with extended-spectrum beta-lactamase-producing
Strength and limitations of this study

- We used a dynamic transmission model to take into account that the risk of colonisation in the intensive care unit depends on the number of extended-spectrum beta-lactamase-producing Enterobacteriaceae carriers and could change over time.

- Parameters used in the model were derived from recent multicentre studies.

- We undertook sensitivity analyses to show the impact of uncertainty in parameter estimation and the impact of model assumptions on the conclusions.

- Directhealthcare worker (HCW) to HCW transmissions as well as environmental contamination were not included in the model.

Enterobacteriaceae (ESBL-PE) has increased worldwide. $^{1-4}$ In Europe, in 2014, the percentage of Escherichia coli and Klebsiella pneumoniae resistant to third-generation cephalosporins in invasive isolates was $12 \%$ and $23 \%$, respectively. A similar trend was observed in the USA, although with large variations between states. ${ }^{2}$

In hospital settings, ESBL-PE acquisition is mainly due to indirect transmission between patients with the hands of healthcare workers (HCWs) as vectors. ${ }^{5}$ Increased prevalence of colonisation augments the risk of acquiring ESBL-PE infection. ${ }^{6}$ Such infections represent a serious socioeconomic burden and are associated with a raised mortality, more frequent hospital admissions in comparison with non-carriers and additional costs. ${ }^{7}$

Many interventions have been proposed to limit the transmission of multidrug-resistant organisms (MDROs) in hospitals. They can be classified as either (1) a 'universal' 
or 'horizontal' approach, applied to all patients, for example, improvement of hand hygiene ( $\mathrm{HH})$ among HCWs or antibiotic stewardship or (2) a 'targeted' or 'vertical' approach, for example, screening and isolation of asymptomatic carriers in addition to infected patients, with the aim of identifying carriers and implementing measures to prevent the transmission from carriers to other patients. $^{8}$

There is general agreement that $\mathrm{HH}$ reduces the transmission of MDROs, especially methicillin-resistant Staphylococcus aureus (MRSA). ${ }^{6}$ However, few studies have evaluated the impact of $\mathrm{HH}$ on the prevention of ESBL-PE dissemination and they have provided conflicting results. ${ }^{9} 10$ The effectiveness of targeted measures in controlling the spread of MDROs, and especially ESBL-PE, remains controversial. This approach is mainly recommended in high-risk units, for example, intensive care units (ICUs). ${ }^{11}$

The implementation of interventions with demonstrated effectiveness in reducing ESBL-PE infections is associated with costs that are generally supported by hospitals. However, when evaluating implementation of an infection prevention programme, one should also take into account savings associated with these interventions, but this has been largely ignored in previous studies.

In this study, we used a mathematical model to evaluate the effectiveness and cost-effectiveness of universal and targeted control strategies for the prevention of ESBL-PE transmission in an ICU in a high-income country.

\section{METHODS \\ Model}

We extended a previously described stochastic, compartmental and dynamic model of ESBL-PE transmission ${ }^{12}$ to assess the economic impact of infection control strategies implemented in a hypothetical, ICU setting. We run the model over a 1 year to capture all costs and health effects relevant to implemented control strategies.

The model simulated the spread of ESBL-PE among patients through contacts with HCWs in an ICU, taking into account hospital admissions and discharges of patients, antibiotic exposure and control interventions (figure 1). The online supplementary text $\mathrm{S} 1$ provides details of the model and its assumptions.

\section{Model simulations and outcomes}

Simulations of the model were performed using Gillespie's method and programmed in $\mathrm{C}++$ language. The outcomes (cases of ESBL-PE transmission, infections, cost of intervention, cost of infections) were calculated after a period of 1 year and averaged over the 1000 Monte Carlo simulations. Cost-effectiveness analysis and graphics were performed in $\mathrm{R}^{13}$

\section{Base case scenario}

In the base case scenario, with no control intervention, we considered that compliance with $\mathrm{HH}$ before/after contact with a patient was $55 \% / 60 \%$, respectively, ${ }^{14}$ and $56 \%$ of patients received antibiotics at ICU admission. ${ }^{15}$

\section{Infection control strategies \\ Universal approaches}

We evaluated control strategies implemented in all patients (independently of their colonisation status) who comprised one or both of the following interventions: (1) improved compliance with $\mathrm{HH}$ and (2) antibiotic stewardship. For HH, we considered different levels of compliance. First, compliance with HH before/after contact with a patient was improved from $55 \% / 60 \%$ at baseline to $55 \% / 80 \%$ or $80 \% / 80 \%$. Second, antibiotic stewardship resulted in halving the proportion of patients on antibiotics at ICU admission and in reducing by $25 \%$ the duration of antibiotic treatment.

\section{Targeted approaches}

We evaluated two strategies that combined screening of patients for ESBL-PE at ICU admission and one of the following interventions implemented: (1) contact precautions (improved compliance with $\mathrm{HH}$ before/after contact with carriers to $80 \% / 80 \%$ ) or (2) cohorting of ESBL-PE carriers with dedicated HCWs. HH compliance for other patients was maintained at baseline level.

\section{Mixed approaches}

We evaluated two strategies combining the targeted approaches with antibiotic stewardship.

\section{Model parameters}

Model parameters and their values are presented in online supplementary table 1.

Based on recent French data, we assumed that $15 \%$ of patients were colonised with ESBL-PE at ICU admission. ${ }^{16}$

Infection status was not included in the model, so we estimated the number of infections by multiplying the cumulated number of colonised patients after 1 year by the probability of developing an infection during an ICU stay, set at $16.4 \% \cdot{ }^{17}$ Even though this value came from a recent large multicentre study, we also considered the impact of lower $(8 \%)$ and higher $(30 \%)$ probability of infection in alternative analyses.

\section{Costs}

The analysis was performed from a public hospital perspective. Cost estimates are based on values reported in euros from 2015 (€1=US\$0.94). We considered the following costs in the model: (1) costs of intervention (material resources and personnel costs) and (2) costs of ESBL-PE infections. The cost of an ESBL-PE infection was estimated using the ESBL-PE-attributable LOS and the cost of a hospital bed-day for infected patients. ${ }^{16} 1819$ See table 1 for cost parameters and online supplementary text $\mathrm{S} 1$ for more details.

\section{Cost-effectiveness evaluation}

To conduct the cost-effectiveness, ${ }^{20}$ we estimated the costs associated with each intervention implemented, 
(A)

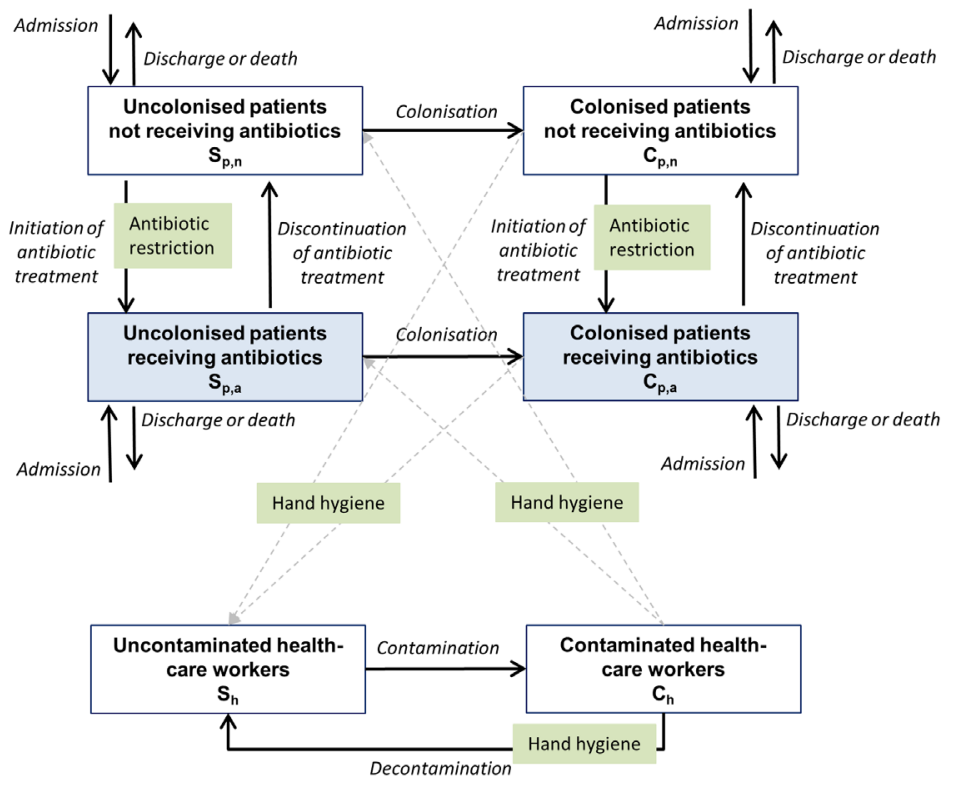

(B)

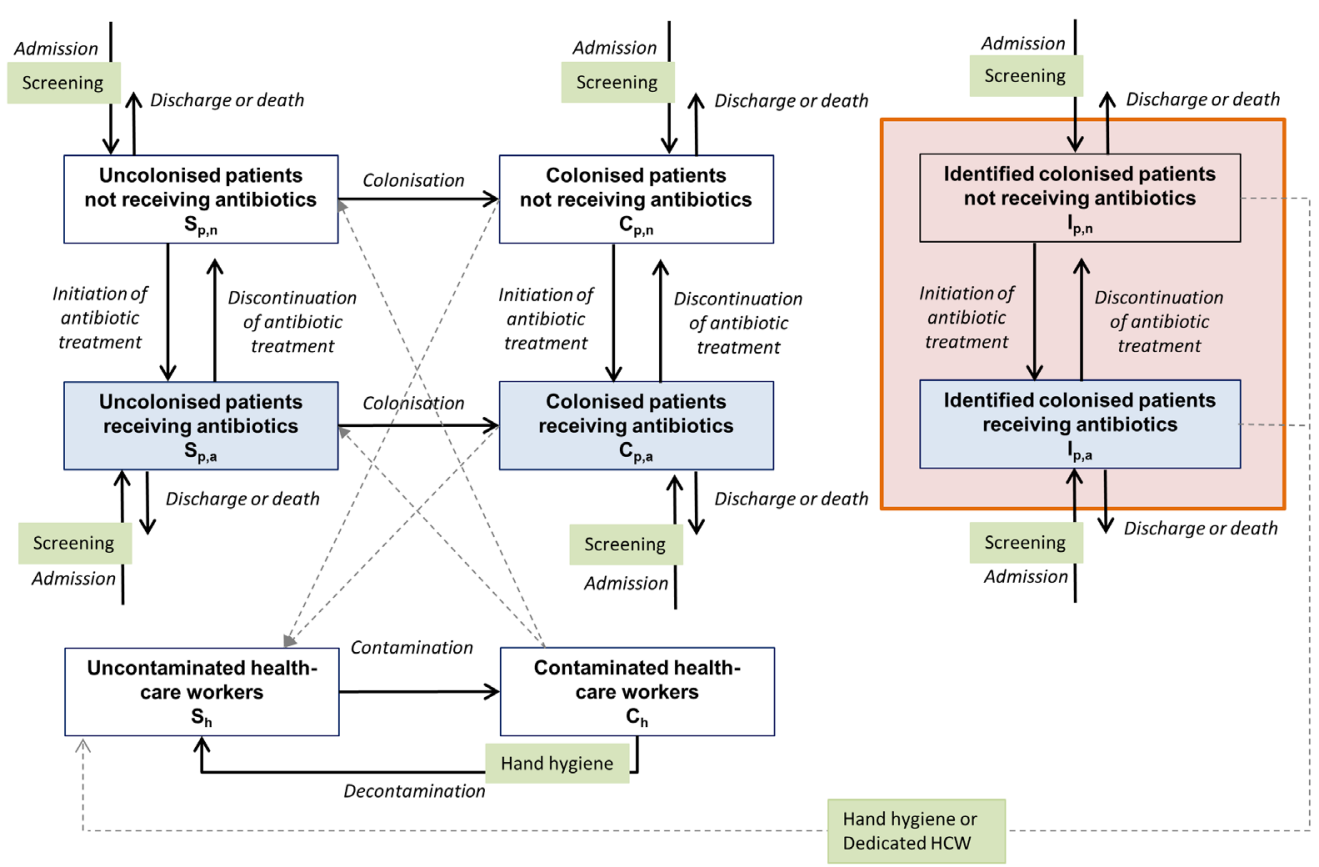

Figure 1 Model of transmission of extended-spectrum beta-lactamase-producing Enterobacteriaceae (ESBL-PE) between patients through contacts with healthcare workers (HCWs) and impact of infection control measures in the transmission process. Solid lines represent the transitions between population groups and dashed lines represent the transmission between patients and HCWs. (A) Impact of universal (horizontal) control measures: (1) hand hygiene (HH) (reduces the transmission among patients and HCWs); (2) antibiotic restriction (reduces the risk of transmission from colonised patients receiving antibiotics to HCWs or from contaminated HCWs to uncolonised patients receiving antibiotics). (B) Impact of targeted (vertical) control measures: screening of patients on intensive care unit admission and identification of patients who had positive screening results (patients surrounded be a shaded box). Implementation of: (1) contact precautions (HH reduces the transmission from identified ESBL-PE carriers to HCWs); (2) cohorting of identified ESBL-PE carriers and attribution of a dedicated HCW (prevents the transmission from cohorted patients to other HCWs and patients). Note that we included two categories of colonised patients: (1) who had false-negative admission screening results and (2) who had positive admission screening results (patients surrounded by a shaded box). 


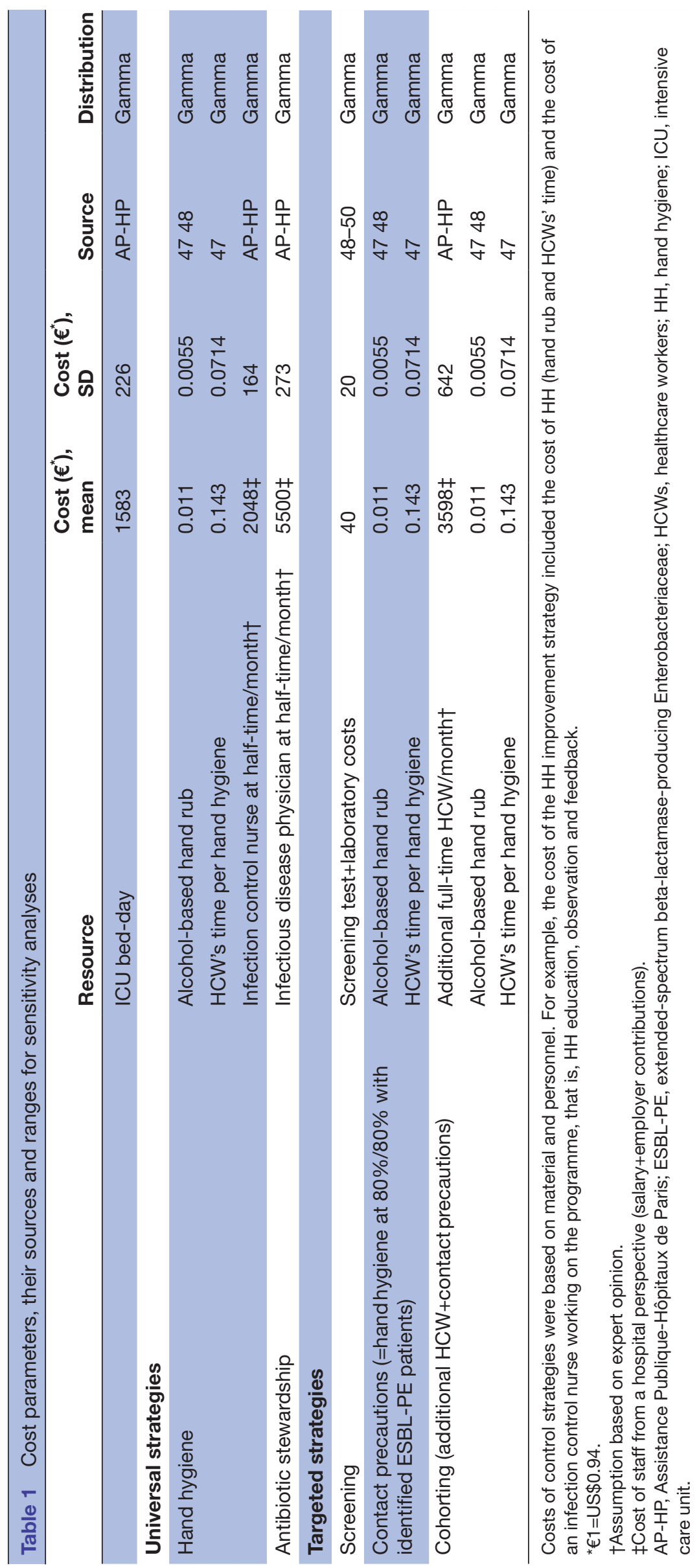

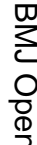

离

$\frac{\bar{c}}{\frac{\mathrm{c}}{\mathrm{D}}}$ 
and the health benefits were related to the number of avoided cases of ESBL-PE infections. First, we determined whether any strategy was dominated by another in terms of costs and health benefits. Second, we determined whether any strategy was dominated through principles of extended dominance (ie, whether the incremental cost-effectiveness ratios (ICERs) decrease as the strategies increases in $\left.\operatorname{cost}^{2021}\right)$. Finally, for the non-dominated strategies, we calculated the incremental cost per case of infection avoided, which is the ratio of the difference in costs between two strategies to the difference in health benefits. This process produces an 'efficient frontier' indicating more costly, but more effective strategies.

\section{Sensitivity analysis}

We performed supplementary analyses to assess the impact of parameter uncertainty on the model's predictions. We first ran a univariate sensitivity analysis to evaluate the cost-effectiveness of strategies in settings with either low or high prevalence of patients colonised at admission (from $5 \%$ to $50 \%$ ). We also considered the impact of a lower $(8 \%)$ and a higher $(30 \%)$ probability of infection in colonised patients. We then investigated the model assuming (1) a lower baseline compliance with HH $(20 \% / 40 \%$ or $40 \% / 50 \%)$, (2) a lower sensitivity of the screening method used to detect ESBL-PE carriers at ICU admission, and (3) a lower, $30 \%$ reduction in antibiotic prescribing.

We also performed an analysis to explore the uncertainty in human time required in an $\mathrm{HH}$ programme and its potential effects. In this analysis, we varied the time an infection control nurse works on the programme (quarter-time, half-time or full-time) simultaneously with the level of $\mathrm{HH}$ compliance achieved (from $55 \% / 60 \%$ to $80 \% / 80 \%$ before after contact).

Finally, we performed a probabilistic sensitivity analysis to explore the effect of joint uncertainty across parameters on the cost-effectiveness of universal versus targeted strategies. We varied the following parameters concurrently: (1) number of HCW contacts with patients, (2) transmission parameters, (3) length of stay of ICU patients, (4) natural decontamination rate for HCW, (5) antibiotic initiation rate, (6) prevalence of ESBL-PE carriage among patients admitted to the ICU, (7) death rate of patients, (8) probability of infection in colonised patients and (9) cost parameters. We randomly sampled values from each of the parameter distributions and calculated the mean costs and mean number of infections for each strategy (averaged over 1000 simulations).

\section{RESULTS}

In the absence of control interventions (base case strategy), 15 new acquisitions (ie, transmissions) and five infections due to ESBL-PE (those from new acquisitions and in patients colonised at admission) occurred per 100 admissions. Compared with the base case (strategy 1 ), all strategies reduced ESBL-PE acquisition and infections within 1 year (figure 2).

Among universal strategies, HH compliance improvement to $80 \% / 80 \%$ (strategy 2) was the most effective, resulting in a mean reduction to 2.9 acquired infections per 100 admissions. Among targeted strategies, screening of patients on admission and cohorting of carriers (strategy 6) was the most effective strategy and resulted in a mean reduction to 2.8 infections per 100 admissions. Screening followed by contact precautions (strategy 5) was the least effective in comparison with all other options. Adding antibiotic stewardship to HH or targeted strategies only slightly improved their effectiveness.

\section{Cost-saving analysis}

In table 2, we present the estimated costs and outcomes over 1 year for all strategies. The mean total cost associated with the base case strategy was estimated at $€ 105$ $344 / 100$ admissions, $€ 94792$ of which was related to infections and $€ 10552$ to interventions. Investments in infection prevention was always cost saving because they avoided cases of ESBL-PE infections and thus costs associated with these infections. For instance, when HH compliance was improved to $80 \% / 80 \%$, the mean cost of the strategy implementation increased to $€ 25639 / 100$ admissions, but the costs related to infections decreased to $€ 54916$, resulting in an overall monetary benefit of $€ 24788 / 100$ admissions in comparison with the base case. This strategy was associated with the highest savings within all evaluated strategies.

\section{Cost-effectiveness analysis}

$\mathrm{HH}$ compliance improvement to $80 \% / 80 \%$ was the least expensive strategy. However, two strategies required higher investments than the $\mathrm{HH}$ programme, but also improved health benefits. To help choose between strategies, we calculated the ICER (figure 3 ). The ICER of $\mathrm{HH}$ improvement to $80 \% / 80 \%$ and antibiotic stewardship (strategy 7) versus HH compliance improvement to $80 \% / 80 \%$ was estimated at $€ 49055 /$ avoided infection (table 2). The ICER of screening, cohorting and antibiotic stewardship (strategy 10) versus HH improvement to $80 \% / 80 \%$ and antibiotic stewardship was estimated at $€ 61$ 994/avoided infection. Other strategies were dominated (more expensive and less effective).

\section{Sensitivity analysis}

Findings from sensitivity analysis showed the robustness of predictions to: (1) the lower/higher prevalence of ESBL-PE carriage on ICU admission, (2) the lower/ higher probability of infections in colonised patients, (3) the baseline compliance with $\mathrm{HH}$ lower than in our core analysis $(20 \% / 40 \%$ or $40 \% / 50 \%)$, (4) the lower sensitivity to detect ESBL-PE carriers at ICU admission and (5) the $30 \%$ reduction in antibiotic prescribing. Results of this analysis are shown in online supplementary text S2, and supplementary figures 1 and 2 supplementary tables $1-4$. 
A

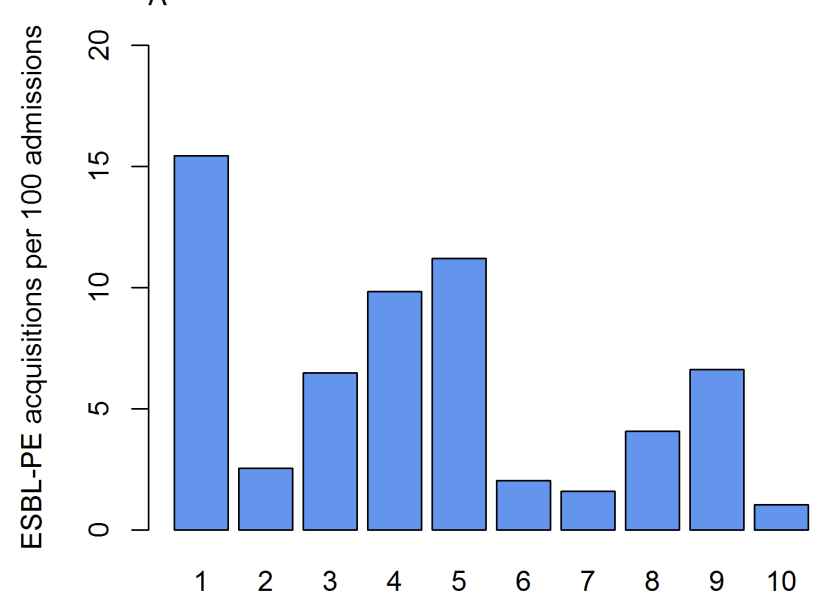

B

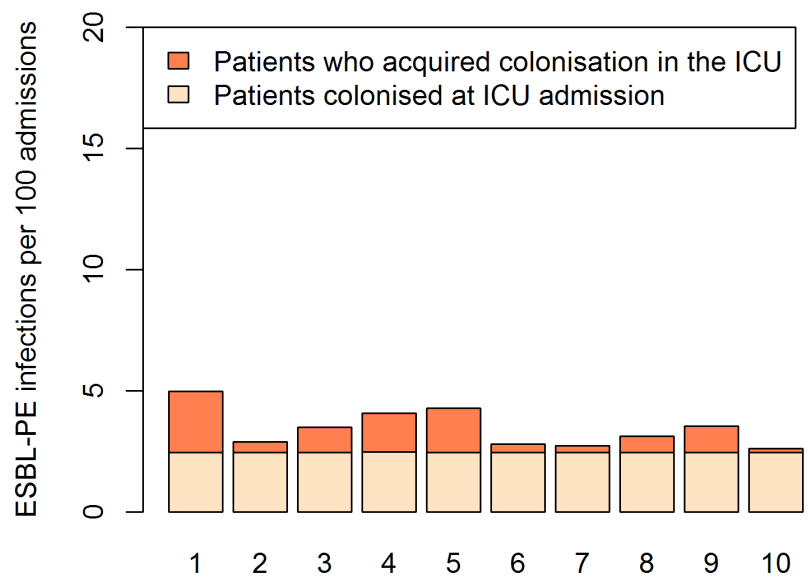

Figure 2 Patient outcomes after 1 year under the different control strategies tested. (A) New acquisitions (transmissions) of extended-spectrum beta-lactamase-producing Enterobacteriaceae (ESBL-PE) per 100 admissions. (B) Total number of ESBLPE infections per 100 admissions in patients who: (1) acquired colonisation in the intensive care unit (ICU) and (2) those who were already colonised at ICU admission. Strategies are: (1) base case (reference strategy with no control intervention and hand hygiene compliance of 55\%/60\% before/after patient contact); (2) hand hygiene $(\mathrm{HH}) \mathrm{improvement} \mathrm{to} 80 \% / 80 \%$; (3) $\mathrm{HH}$ improvement to 55\%/80\%; (4) antibiotic reduction; (5) screening of all admissions and contact precautions for identified carriers;

(6) screening of all admissions and cohorting of identified carriers; (7) $\mathrm{HH}$ improvement to $80 \% / 80 \%$ and antibiotic reduction; (8) $\mathrm{HH}$ improvement to $55 \% / 80 \%$ and antibiotic reduction; (9) screening of all admissions, contact precautions with identified carriers and antibiotic reduction; (10) screening of all admissions, cohorting of identified carriers and antibiotic reduction.

In a second sensitivity analysis, we focused on human time and performance to improve $\mathrm{HH}$ compliance. If an infection control nurse was assumed to work quarter-time, half-time or full-time on the programme, the HH compliance had to increase by at least $5 \%, 7.5 \%$ or $15 \%$, respectively, to make the programme cost saving compared with the base case (online supplementary table S5A).

In comparison with the screening and cohorting strategy, the $\mathrm{HH}$ improvement was cost saving when an infection control nurse worked quarter-time or halftime on the programme, and $\mathrm{HH}$ compliance increased by at least $12.5 \%$ or $17.5 \%$, respectively. The screening and cohorting strategy dominated the $\mathrm{HH}$ improvement programme when an infection control nurse was working full-time on the programme (online supplementary table S5B).

Finally, the probabilistic sensitivity analysis showed that improvement of $\mathrm{HH}$ to $80 \% / 80 \%$ (strategy 2) was less expensive than the screening and cohorting intervention (strategy 6) in 91\% of simulations. Among them, in $42 \%$ of simulations, the HH strategy was less expensive but more effective (dominated the strategy 6 ), and in $49 \%$ of runs the screening and cohorting was more effective and more expensive (online supplementary figure S3). Screening and contact precautions (strategy 5) were always less effective than improvement of $\mathrm{HH}$ to $80 \% / 80 \%$ (strategy 2) (online supplementary figure $\mathrm{S} 4$ ).

\section{DISCUSSION}

The impact of infection control strategies for preventing ESBL-PE transmission is controversial because clinical studies cannot account for the multiple confounding factors, notably both infection control measures and antibiotic stewardship. Despite several recent high-level interventional studies (Climo et al, ${ }^{22}$ Derde et al, ${ }^{9}$ Huang et $\left.a l^{23}\right)$, the most effective and cost-effective interventions for controlling MDROs are still debated. Since the spread of ESBL-PE between patients is a dynamic and complex process, modelling can help for understanding the transmission mechanisms and deciding which intervention are to be preferred (Doan et al, ${ }^{24}$ Grundmann and Hellriegel $^{25}$ ).

Our model estimated the annual burden of ESBL-PE infections in a French ICU at $€ 94792$ per 100 admissions in the base case strategy. Several prior studies have reported the cost of infections due to MDROs in the ICU. ${ }^{26-29}$ However, even though all authors underlined the high costs of infections, comparison between studies remains difficult. Estimated costs varied according to the country, but also to the population studied, for example, patients with site-specific or micro-organism-specific infections. Moreover, the methods used to estimate the costs were not similar in all publications.

In recent years, mathematical models have increasingly been used to study the cost-effectiveness of control strategies. For example, Robotham et a $\vec{l}^{30}$ compared a 


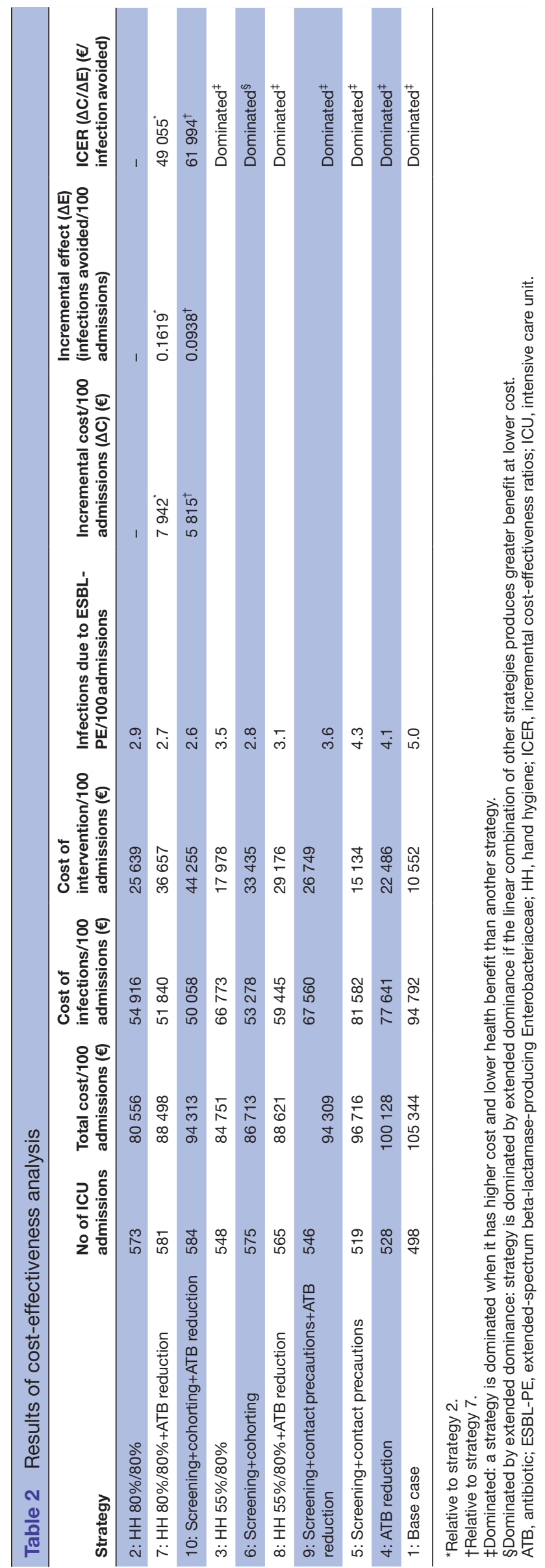

wide range of strategies to control MRSA transmission in ICUs and found that universal decolonisation was the most cost-effective option. In another study, Gidengil et $a l^{31}$ compared hospital strategies to prevent MRSA transmission and infections in an ICU. They confirmed that universal decolonisation was the most cost saving.

While decolonisation regimens have been indicated as cost-effective for MRSA, only a few studies have examined the effect of decolonisation on ESBL-PE carriage. ${ }^{32} 33$ These studies have shown that decolonisation strategies might be efficacious only in the short term. Moreover, they have reported the risk of emergence of resistance to antibiotics used for decolonisation, namely to colimycin, which is the last-line effective therapy against carbapenemase-producing Enterobacteriaceae. ${ }^{33}$ Thus, decolonisation was not considered in our study.

Our study is the first to compare the effectiveness and the costs of universal and targeted control strategies in the context of the spread of ESBL-PE in ICUs. Our model predicted that improving $\mathrm{HH}$ to $80 \% / 80 \%$ in contacts with all patients would prevent $83 \%$ of ESBL-PE acquisitions and avoid at least two out of five infections per 100 admissions. This strategy represented the most cost saving, with a monetary benefit of $€ 24788$ per 100 admissions.

The association between $\mathrm{HH}$ and reduction of MDROs infections has long been known and $\mathrm{HH}$ has been accepted as a crucial component of infection prevention. ${ }^{34} \mathrm{HH}$ has in addition the benefit of being effective for reducing transmission of many resistant or susceptible bacteria. ${ }^{34} \mathrm{~A}$ recent publication reported that a programme designed to control MRSA by implementing universal components in addition to screening and contact precautions for MRSA carriers also effectively reduced the incidence of resistant gram-negative bacteria, the most likely being ESBL-PE. ${ }^{10}$ Thus, an $\mathrm{HH}$ programme designed to reduce ESBL-PE transmission may have positive effects on reducing the transmission of other micro-organisms, and the overall economic benefit of an $\mathrm{HH}$ programme for the hospital might be greater than reported in our study.

Despite the confirmed effectiveness of $\mathrm{HH}$ and national and international recommendations, compliance with $\mathrm{HH}$ remains low and is often lower than values used in our base case analysis. ${ }^{35}{ }^{36}$ Furthermore, improving $\mathrm{HH}$ compliance from $60 \%$ to $80 \%$ may be far more difficult and costly, challenging than improving from lower baseline level. However, we showed in a sensitivity analysis that improving $\mathrm{HH}$ remained the most cost-saving strategy even in a low baseline compliance scenario. Different strategies have been suggested to improve $\mathrm{HH}$ in hospitals, ${ }^{37}$ but the evidence-based approach is still lacking. Recently, a review ${ }^{38}$ concluded that a multimodal strategy proposed by WHO and consisting of five components: (1) system change, (2) training and education, (3) observation and feedback, (4) reminders in the hospital and (5) a hospital safety climate, was effective at increasing $\mathrm{HH}$ among HCWs. Moreover, the authors underlined that additional measures (eg, reward incentives for reaching a certain level of compliance) could lead to further improvements. 


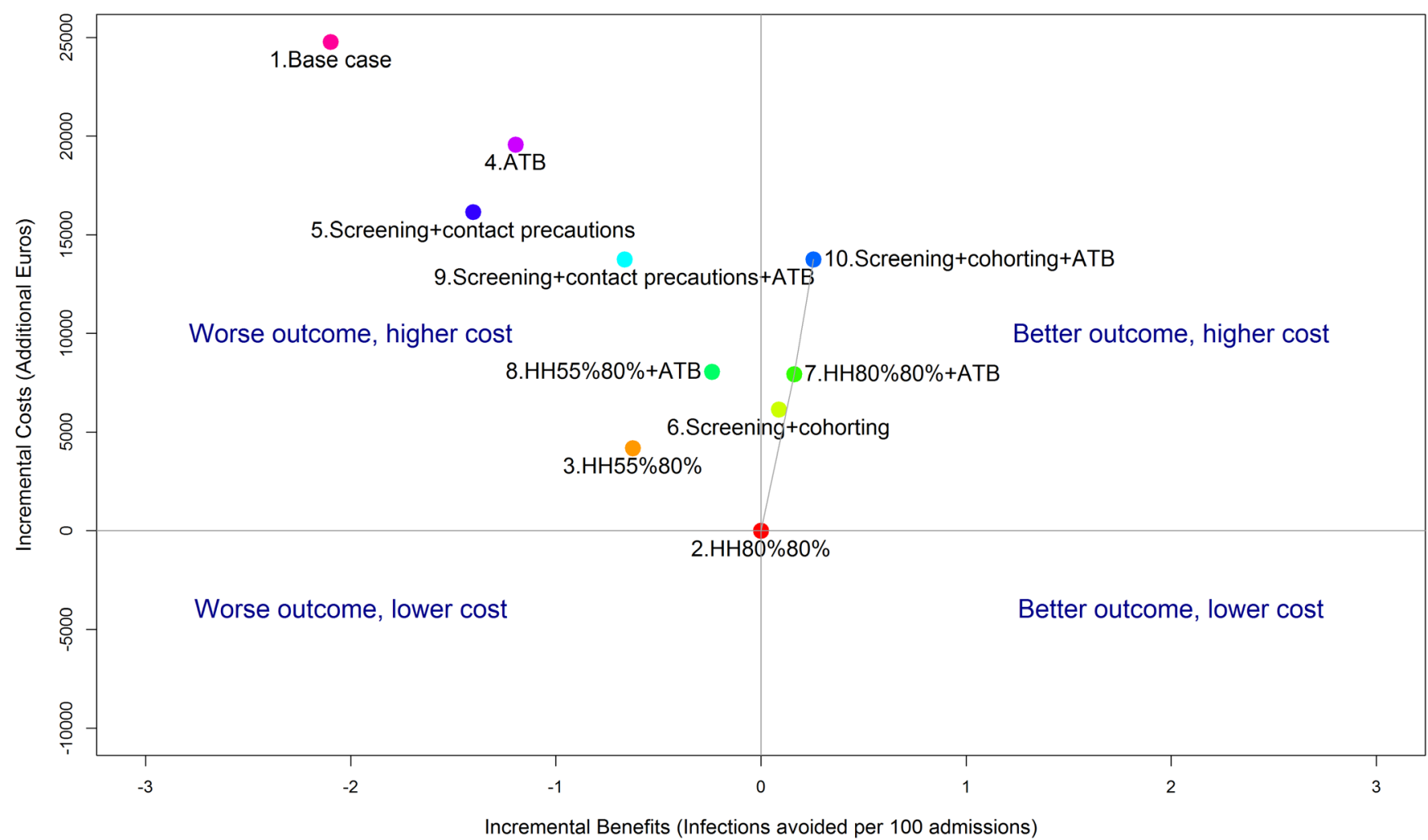

Figure 3 Cost-effectiveness plane showing the incremental health benefits (infections avoided) and costs relative to the least expensive strategy (strategy 2). Strategies 1, 3, 4, 5, 8, 9 are dominated as they have both a worse outcome and a higher cost. The efficiency frontier (grey line), joins strategy 2 with more expensive and more efficient strategies (7 and 10). Strategy 6 is extended to this frontier and excluded by the principle of extended dominance. The slope of the efficiency frontier represents the incremental cost-effectiveness. Strategies are: (1) base case (reference strategy with no control intervention and hand hygiene $(\mathrm{HH}$ ) compliance of 55\%/60\% before/after patient contact); (2) HH improvement to $80 \% / 80 \%$; (3) HH improvement to $55 \% / 80 \%$; (4) antibiotic (ATB) reduction; (5) screening of all admissions and contact precautions with identified carriers; (6) screening of all admissions and cohorting of identified carriers; (7) $\mathrm{HH}$ improvement to $80 \% / 80 \%$ and antibiotic reduction; (8) $\mathrm{HH}$ improvement to $55 \% / 80 \%$ and antibiotic reduction; (9) screening of all admissions, contact precautions with identified carriers and antibiotic reduction; (10) screening of all admissions, cohorting of identified carriers and antibiotic reduction.

In our study, we assumed that a key component of an $\mathrm{HH}$ programme was a dedicated staff working on the programme (ie, HH education, observation and feedback). We hypothesised, for example, that to improve $\mathrm{HH}$ compliance an infection control nurse working half-time would be sufficient. However, this assumption was based on expert opinion; we performed a sensitivity analysis to explore the uncertainty of the required time dedicated to the $\mathrm{HH}$ programme and its expected effects.

Screening strategies have been used to prevent transmission of MDROs; however, in a sensitivity analysis, we showed that improvement of $\mathrm{HH}$ to $80 \% / 80 \%$ was always more effective than screening and contact precautions and mostly less expensive than the screening and cohorting intervention. However, we can hypothesise that in the case of highly resistant bacteria (eg, carbapenem-resistant Enterobacteriaceae) where there is a highest clinical impact on the outcomes of infected patients, given the lack of therapeutic options, a rapid identification and cohorting of carriers may be more beneficial from the hospital but also societal perspective.

Antibiotic use is the major driver for the selection of antibiotic-resistant bacteria ${ }^{39}$ and many strategies have been proposed to reduce the use of antibiotics in hospitals. ${ }^{40}$ These strategies could be implemented and associated with different efficacies and costs. ${ }^{41}$ Here, we considered that antibiotic stewardship, based on the introduction of an infectious disease specialist to the ward, led to a $50 \%$ reduction in antibiotic use. ${ }^{42}$ However, despite this optimistic scenario, we found that antibiotic stewardship was less effective than $\mathrm{HH}$ or a screening and cohorting strategy.

Under the hypotheses used in our model, we also demonstrated in a previous study through sensitivity analyses that antibiotic parameters did not significantly influence the effectiveness of interventions. ${ }^{12}$

However, adding antibiotic stewardship to an $\mathrm{HH}$ strategy slightly improved its effectiveness and may be 
worthy of consideration if the decision-makers are willing to pay at least $€ 49055$ per infection avoided (we calculated that it would be equivalent to $€ 5562$ per life-year gained (LYG)). Combining antibiotic stewardship with screening and cohorting was even more effective than combining $\mathrm{HH}$ and antibiotic stewardship, but with an additional cost of $€ 61994$ per infection avoided (or $€ 7$ 030/LYG).

Our study has several strengths. First, we used a dynamic model to represent interactions between patients and HCWs and to take into account that the risk of colonisation in the ICU depends on the number of ESBL carriers and could change over time. Moreover, our model incorporated the key elements of ESBL-PE transmission, such as the impact of prevalence at admission or antibiotic treatment. Second, we used input parameters derived from recent multicentre studies. Third, we estimated the cost of HCW according to the time they spend working on the programme based on the best evidence from the literature and expert opinion. Finally, we assessed the impact of uncertainty in parameter estimation and the impact of model assumptions on the model's predictions by performing multiple sensitivity analyses.

Our study also has several limitations. ICU parameters and costs were based mostly on French data, and ESBL-PE infections, prevalence, compliance with control measures and costs may be different in other countries.

A recent multicentre cohort study ${ }^{17}$ found no difference in LOS between infected and colonised patients. Thus, in order to simplify assumptions, the 'infected' state was not included to the model. However, infected patients are potentially more contaminating HCW hands, disseminating the organism in the environment and increase the transmissibility. ${ }^{43}$ Thus, consequently we may have underestimated the number of acquisitions in the ICU and the impact of control measures.

The epidemiological characteristics of ESBL-PE are complex and may vary, depending on ESBL-PE species. For example, Thiébaut $e t a l^{44}$ showed that $E$. coli ESBL was mainly imported $(66 \%)$ and $K$. pneumoniae ESBL was acquired (77\%). Furthermore, the differential capacity of cross-transmission between ESBL E. coli and other Enterobacteriaceae has been clearly established. ${ }^{45}$ In a previous publication from our group, ${ }^{12}$ however, we showed no difference in the effectiveness of control measures, whatever the Enterobacteriaceae considered, either E. coli or another Enterobacteriaceae. We therefore decided to consider Enterobacteriaceae globally, a situation that can be extended to carbapenemase-producing Enterobacteriaceae.

We modelled an ICU as a single-room unit where transmission among patients results via contacts with HCWs. In the absence of detailed information on transmission of ESBL-PE in hospital wards, we ignored direct HCW-to-HCW transmissions as well as environmental contamination or excreta management.

ESBL-PE acquisition in the ICU can lead to transmission from an ICU-acquired case and infection in downstream units, thus increasing costs of hospitalisation. Moreover, colonisation with ESBL-PE may persist several months after hospital discharge ${ }^{46}$ therefore increasing the risk of infection with potential subsequent treatment failure. Thus, an efficient intervention to prevent the inhospital cross-transmission may also have an impact on the prevention of postdischarge infections and the need for readmissions.

Our cost evaluation therefore underestimated health benefits and cost savings resulting from inhospital interventions to control ESBL-PE, but participate to demonstrate the usefulness of inhospital intervention to prevent further costs.

\section{CONCLUSION}

Our study suggests that a universal approach with improved compliance with $\mathrm{HH}$ was the most cost-saving strategy to prevent the transmission of ESBL-PE in an ICU setting. Screening and cohorting of carriers had comparable effectiveness to $\mathrm{HH}$ improvement, but was more expensive.

Antibiotic stewardship was not cost-effective in comparison with other options. However, adding antibiotic restriction to the $\mathrm{HH}$ or the screening and cohorting strategies slightly improved their effectiveness and may be worthy of consideration by decision-makers.

\section{Author affiliations}

${ }^{1}$ IAME, UMR 1137, INSERM, Paris, France

${ }^{2}$ University of Paris Diderot, Sorbonne Paris Cité, Paris, France

${ }^{3}$ Infection Control Unit, Bichat-Claude Bernard Hospital, AP-HP, Paris, France ${ }^{4}$ Bacteriology Laboratory, Bichat-Claude Bernard Hospital, AP-HP, Paris, France

${ }^{5}$ Pierre Louis Institute of Epidemiology and Public Health (IPLESPUMRS 1136), INSERM, UPMC University Paris 06, Sorbonne University, Paris, France

${ }^{6}$ Infectious and Tropical Diseases Department, Bichat-Claude Bernard Hospital, AP-HP, Paris, France

Acknowledgements We thank Laurence Armand for useful discussion on our study.

Contributors YY, J-CL, CP and LKS designed the study. YY, J-CL, P-YB, AA, CP and LKS contributed to the development of the model. CP, AP, GB, ER and LKS collected the data. CP and LKS wrote the code. LKS conducted computer simulations and result analysis. LKS, J-CL and YY drafted the manuscript. All authors read and critically revised the manuscript.

Funding This work was supported by the French government (PREPS program (grant number 13-0693)) and by the National Institute for Health and Medical Research (INSERM).

Competing interests None declared.

Provenance and peer review Not commissioned; externally peer reviewed.

Data sharing statement Details of the computer code for the model are available from the corresponding author.

Open Access This is an Open Access article distributed in accordance with the Creative Commons Attribution Non Commercial (CC BY-NC 4.0) license, which permits others to distribute, remix, adapt, build upon this work non-commercially, and license their derivative works on different terms, provided the original work is properly cited and the use is non-commercial. See: http://creativecommons.org/ licenses/by-nc/4.0/

(C) Article author(s) (or their employer(s) unless otherwise stated in the text of the article) 2017. All rights reserved. No commercial use is permitted unless otherwise expressly granted. 


\section{REFERENCES}

1. European Centre for Disease Prevention and Control (ECDC). Surveillance reports: antimicrobial resistance surveillance in Europe, 2014. http://ecdc.europa.eu/en/publications/publications/ antimicrobial-resistance-europe-2014.pdf (accessed 30 Sep 2016).

2. Centers for Disease Control and Prevention (CDC). Antibiotic resistance patient safety atlas. http://gis.cdc.gov/grasp/PSA/ Downloads/ARPatientSafetyAtlas-SummaryofResults.pdf (accessed 30 Sep 2016).

3. Sidjabat HE, Paterson DL. Multidrug-resistant Escherichia coli in Asia: epidemiology and management. Expert Rev Anti Infect Ther 2015;13:575-91.

4. Tansarli GS, Poulikakos P, Kapaskelis A, et al. Proportion of extended-spectrum $\beta$-lactamase (ESBL)-producing isolates among Enterobacteriaceae in Africa: evaluation of the evidence-systematic review. J Antimicrob Chemother 2014;69:1177-84.

5. Paterson DL, Bonomo RA. Extended-spectrum beta-lactamases: a clinical update. Clin Microbiol Rev 2005;18:657-86.

6. World Health Organization. Evidence of hand hygiene to reduce transmission and infections by multi-drug resistant organisms in health-care settings. http://www.who.int/gpsc/5may/MDRO literature-review.pdf (accessed 7 Sep 2016).

7. Gandra S, Barter DM, Laxminarayan R. Economic burden of antibiotic resistance: how much do we really know? Clin Microbiol Infect 2014;20:973-80

8. Septimus E, Weinstein RA, Perl TM, et al. Approaches for preventing healthcare-associated infections: go long or go wide? Infect Control Hosp Epidemiol 2014;35(Suppl 2):S10-14.

9. Derde LPG, Cooper BS, Goossens H, et al. Interventions to reduce colonisation and transmission of antimicrobial-resistant bacteria in intensive care units: an interrupted time series study and cluster randomised trial. Lancet Infect Dis 2014;14:31-9.

10. Goto M, O'Shea AMJ, Livorsi DJ, et al. The Effect of a Nationwide Infection Control Program Expansion on Hospital-Onset Gramnegative Rod Bacteremia in 130 Veterans Health Administration Medical Centers: an interrupted time-series analysis. Clin Infect Dis 2016;63:642-50.

11. Otter JA, Mutters NT, Tacconelli E, et al. Controversies in guidelines for the control of multidrug-resistant Gram-negative bacteria in EU countries. Clin Microbiol Infect 2015;21:1057-66.

12. Pelat $\mathrm{C}$, Kardaś-Słoma L, Birgand G, et al. Hand Hygiene, Cohorting, or Antibiotic Restriction to Control Outbreaks of Multidrug-Resistant Enterobacteriaceae. Infect Control Hosp Epidemiol 2016;37:272-80.

13. R Core Team. R. The R project for statistical computing. https://www. r-project.org/ (accessed 15 Sep 2016).

14. Venier AG, Zaro-Goni D, Pefau M, et al. Performance of hand hygiene in 214 healthcare facilities in South-Western France. J Hosp Infect 2009;71:280-2

15. REA-Raisin network. Surveillance des infections nosocomiales en réanimation adulte. http://invs.santepubliquefrance.fr/Publicationset-outils/Rapports-et-syntheses/Maladies-infectieuses/2012/ Surveillance-des-infections-nosocomiales-en-reanimation-adulte (accessed 15 Sep 2016).

16. Razazi K, Derde LP, Verachten M, et al. Clinical impact and risk factors for colonization with extended-spectrum $\beta$-lactamaseproducing bacteria in the intensive care unit. Intensive Care Med 2012;38:1769-78.

17. Barbier F, Pommier C, Essaied W, et al. Colonization and infection with extended-spectrum $\beta$-lactamase-producing Enterobacteriaceae in ICU patients: what impact on outcomes and carbapenem exposure? J Antimicrob Chemother 2016;71:1088-97.

18. Viau R, Frank KM, Jacobs MR, et al. Intestinal carriage of carbapenemase-producing organisms: current status of surveillance methods. Clin Microbiol Rev 2016;29:1-27.

19. Vasudevan A, Memon BI, Mukhopadhyay A, et al. The costs of nosocomial resistant gram negative intensive care unit infections among patients with the systemic inflammatory response syndromea propensity matched case control study. Antimicrob Resist Infect Control 2015;4:3

20. Briggs A, Claxton K, Sculpher M. Oxford University Press. Decision modelling for health economic evaluation, 2006. https://www.york. ac.uk/che/publications/books/decision-modelling/ (accessed 6 Sep 2016).

21. Cantor SB, Ganiats TG. Incremental cost-effectiveness analysis: the optimal strategy depends on the strategy set. $J$ Clin Epidemiol 1999;52:517-22.

22. Climo MW, Yokoe DS, Warren DK, et al. Effect of daily chlorhexidine bathing on hospital-acquired infection. N Engl J Med 2013;368:533-42.
23. Huang SS, Septimus E, Kleinman K, et al. Targeted versus universal decolonization to prevent ICU infection. $N$ Engl J Med 2013;368:2255-65.

24. Doan TN, Kong DC, Kirkpatrick CM, et al. Optimizing hospital infection control: the role of mathematical modeling. Infect Control Hosp Epidemiol 2014;35:1521-30.

25. Grundmann H, Hellriegel B. Mathematical modelling: a tool for hospital infection control. Lancet Infect Dis 2006;6:39-45.

26. Chaix C, Durand-Zaleski I, Alberti C, et al. Control of endemic methicillin-resistant Staphylococcus aureus: a cost-benefit analysis in an intensive care unit. JAMA 1999;282:1745-51.

27. Roberts RR, Hota B, Ahmad I, et al. Hospital and societal costs of antimicrobial-resistant infections in a Chicago teaching hospital: implications for antibiotic stewardship. Clin Infect Dis 2009;49:1175-84.

28. Mauldin PD, Salgado CD, Hansen IS, et al. Attributable hospital cost and length of stay associated with health care-associated infections caused by antibiotic-resistant gram-negative bacteria. Antimicrob Agents Chemother 2010;54:109-15.

29. Lee SY, Kotapati S, Kuti JL, et al. Impact of extended-spectrum beta-lactamase-producing Escherichia coli and Klebsiella species on clinical outcomes and hospital costs: a matched cohort study. Infect Control Hosp Epidemiol 2006;27:1226-32.

30. Robotham JV, Graves N, Cookson BD, et al. Screening, isolation, and decolonisation strategies in the control of meticillin resistant Staphylococcus aureus in intensive care units: cost effectiveness evaluation. BMJ 2011;343:d5694.

31. Gidengil CA, Gay C, Huang SS, et al. Cost-effectiveness of strategies to prevent methicillin-resistant Staphylococcus aureus transmission and infection in an intensive care unit. Infect Control Hosp Epidemiol 2015;36:17-27.

32. Huttner B, Haustein T, Uçkay I, et al. Decolonization of intestinal carriage of extended-spectrum $\beta$-lactamase-producing Enterobacteriaceae with oral colistin and neomycin: a randomized, double-blind, placebo-controlled trial. J Antimicrob Chemother 2013;68:2375-82.

33. Halaby T, Al Naiemi N, Kluytmans J, et al. Emergence of colistin resistance in Enterobacteriaceae after the introduction of selective digestive tract decontamination in an intensive care unit. Antimicrob Agents Chemother 2013;57:3224-9.

34. World Health Organization. WHO Guidelines on Hand Hygiene in Health Care, 2009. http://apps.who.int/iris/bitstream/10665/44102/1/ 9789241597906_eng.pdf (accessed 6 Sep 2016).

35. Scheithauer S, Haefner H, Schwanz T, et al. Compliance with hand hygiene on surgical, medical, and neurologic intensive care units: direct observation versus calculated disinfectant usage. Am J Infect Control 2009;37:835-41.

36. Cepeda JA, Whitehouse T, Cooper B, et al. Isolation of patients in single rooms or cohorts to reduce spread of MRSA in intensive-care units: prospective two centre study. The Lancet 2005;365:295-304.

37. Pittet D, Hugonnet S, Harbarth S, et al. Effectiveness of a hospitalwide programme to improve compliance with hand hygiene. The Lancet 2000;356:1307-12.

38. Luangasanatip N, Hongsuwan M, Limmathurotsakul D, et al. Comparative efficacy of interventions to promote hand hygiene in hospital: systematic review and network meta-analysis. BMJ 2015;351:h3728.

39. Holmes AH, Moore LS, Sundsfjord A, et al. Understanding the mechanisms and drivers of antimicrobial resistance. Lancet 2016;387:176-87.

40. Davey P, Brown E, Charani E, et al. Interventions to improve antibiotic prescribing practices for hospital inpatients. Cochrane Database Syst Rev 2013:CD003543.

41. de Jong E, van Oers JA, Beishuizen A, et al. Efficacy and safety of procalcitonin guidance in reducing the duration of antibiotic treatment in critically ill patients: a randomised, controlled, openlabel trial. Lancet Infect Dis 2016;16:819-27.

42. Roger P-M, Farhad R, Pulcini C, et al. Elderly patients presenting with fever and respiratory problems in an intensive care unit. Diagnostic, therapeutic and prognostic impact of a systematic infectious disease consultation. Presse Medicale Paris Fr 2003;32:1699-704.

43. Ruppé E, Lixandru B, Cojocaru R, et al. Relative fecal abundance of extended-spectrum- $\beta$-lactamase-producing Escherichia coli strains and their occurrence in urinary tract infections in women. Antimicrob Agents Chemother 2013;57:4512-7.

44. Thiébaut AC, Arlet $\mathrm{G}$, Andremont A, et al. Variability of intestinal colonization with third-generation cephalosporin-resistant Enterobacteriaceae and antibiotic use in intensive care units. J Antimicrob Chemother 2012;67:1525-36. 
45. Tschudin-Sutter S, Lucet J-C, Mutters NT, et al. Contact precautions for preventing nosocomial transmission of ESBL-producing Escherichia coli - a point/counterpoint review. 2017. [Epub ahead of print 3 Apr 2017].

46. Birgand G, Armand-Lefevre L, Lolom I, et al. Duration of colonization by extended-spectrum $\beta$-lactamase-producing Enterobacteriaceae after hospital discharge. Am J Infect Control 2013;41:443-7.

47. Girard R, Aupee M, Erb M, et al. Hand rub dose needed for a single disinfection varies according to product: a bias in benchmarking using indirect hand hygiene indicator. J Epidemiol Glob Health 2012;2:193-8.
48. Kaier K. Economic implications of the dynamic relationship between antibiotic use and hospital-acquired infections. Value Health 2012;15:87-93.

49. Armand L. Place des nouvelles techniques dans l'optimisation diagnostique et thérapeutique des infections nosocomiales en réanimation. http://cclin-sudest.chu-lyon.fr/Reseaux/REA/Journee/ 2014/6 Bacterio NouveauxOutils.pdf (accessed 6 Sep 2016).

50. Birgand G, Ruimy R, Schwarzinger M, et al. Rapid detection of glycopeptide-resistant enterococci: impact on decision-making and costs. Antimicrob Resist Infect Control 2013;2:30. 